\title{
Evaluating the effects of synthetic POM cycles and NAD kinase expression on fatty alcohol production in Saccharomyces cerevisiae
}

\section{Bonnie A McNeil}

University of Alberta

Charfeddine Khalifa

University of Alberta

Anagha Krishnan

University of Alberta

David T Stuart ( $\nabla$ dtstuart@ualberta.ca )

University of Alberta https://orcid.org/0000-0002-0001-7713

\section{Research}

Keywords: Redox balance, transhydrogenase, NADPH, Saccharomyces cerevisiae, metabolic engineering, POM cycle, NAD kinase

Posted Date: January 23rd, 2020

DOI: https://doi.org/10.21203/rs.2.21642/v1

License: (9) This work is licensed under a Creative Commons Attribution 4.0 International License. Read Full License 


\section{Abstract}

Background: NADPH-dependent enzymes play important roles in many anabolic reactions and the availability of redox cofactors can influence metabolic flux ultimately influencing titers of bioproducts produced by engineered microbial cells. This may be especially true of oleochemical production when carbon flux through the highly NADPH-dependent fatty acid biosynthesis pathway is increased. While pathway specific approaches are often applied to counter redox imbalance, a study evaluating generalized approaches to improved NADPH availability is lacking in Saccharomyces cerevisiae .

Results: Here, we have created four unique synthetic Pyruvate-Oxaloacetate-Malate "POM" cycles consisting of either of the endogenous isoforms of pyruvate carboxylase ( PYC1 or PYC2 ), a modified version of malate dehydrogenase ( $\mathrm{MDH} 1$ or 'MDH2 ), and a truncated cytosolic form of the endogenous malic enzyme ( sMAE1 ). Only the POM cycle that combined expression of PYC1, 'MDH2 , and SMAE1 increased the titer of fatty alcohols produced; however, it did so in two unique fatty alcohol producing strains. In a FAS1 overexpression background, expression of this synthetic POM cycle increased fatty alcohol titers by $40 \%$ from $49.0 \pm 2.2 \mathrm{mg} / \mathrm{L}$ to $68.6 \pm 3.3 \mathrm{mg} / \mathrm{L}$ and showed similar results in a zwf 1 deletion strain. The effect of overexpression of the endogenous NAD+ kinases UTR1, YEF1, and a cytosolic version of POS5 were also tested. We found that expression of POS5c resulted in an $~ 35 \%$ increase in fatty alcohol titer, while the overexpression of the UTR1 or YEF1 did not significantly influence titers. In these minimally engineered cells, combined overexpression of PYC1 , 'MDH2 , SMAE1 and POS5c did not further increase titers

Conclusions: Overexpression of PYC1 in conjunction with 'MDH2 and SMAE1 results in a synthetic POM cycle which can be utilized to improve fatty alcohol production in engineered strains of S. cerevisiae . Additionally, overexpression of a truncated version of POS5 ( POS5c) results in similar increases in fatty alcohol production. These findings may serve to provide a generalized mechanism to increase NADPH production in engineered cells, resulting in increased bioproduct titers.

\section{Background}

Metabolic engineering of microbial strains can cause perturbations in metabolite abundance and redoxcofactor availability creating bottlenecks which can hinder cellular growth and negatively influence the titers, rates and yields (TRY) of desired products [1-3]. In particular, upregulation of biosynthetic processes can cause increased demand for the redox cofactor Nicotinamide Adenine Dinucleotide Phosphate (NADP), which in its reduced form (NADPH) plays a vital role in providing the redox potential to drive many anabolic reactions [4]. Therefore, standard cellular biosynthetic processes (such as the generation of lipids, phospholipids, amino acids and nucleic acids) and achieving high titers of biosynthetic products through metabolic engineering rely on the recycling of redox cofactors to NADPH which cells typically achieve through one of the three following ways: (i) from NADP ${ }^{+}$by NADP ${ }^{+}$ dependent enzymes (ii) from NAD ${ }^{+}$or NADH kinases and (iii) by catalyzing the reaction NADH + NADP ${ }^{+}$ $\rightleftarrows \mathrm{NAD}{ }^{+}+\mathrm{NADPH}$ through the activity of transhydrogenases. 
An increased demand for NADPH is particularly salient in the production of fatty acid-based chemicals, as the anabolism of fatty acyl substrates is highly NADPH-dependent. The fatty acid synthase (FAS) complex in S. cerevisiae requires 14 molecules of NADPH for the production of one C16 acyl chain. Additionally, the terminal enzymes employed in many of these conversion processes also rely on NADPH as cofactors, such as is the case in the production of fatty alcohols through the use of NADPH-dependent fatty acyl-CoA reductases (FAR). To-date many approaches have been employed in engineered microbial cells to generate NADPH to fuel biosynthesis and to counter imposed redox-cofactor imbalances with the majority of approaches clustering around three themes: (i) elimination or knockdown of cofactor competitive pathways, (ii) altering cofactor preference of overexpressed enzymes, (iii) and the transformation of NAD to its analog NADP [5]. While the first two approaches can be uniquely targeted to specific production pathways, they alter carbon flux and therefore do not offer a generalized mechanism that can be widely applied to a variety of metabolic engineering projects. However, efficiently transforming NAD to NADP, through the overexpression of transhydrogenases, transhydrogenase-like shunts (also referred to as Pyruvate-Oxaloacetate-Malate or "POM" cycles) and NAD kinases is a general mechanism to improve cellular NADPH levels that can be applied to a wide variety of bioengineering projects without altering the net cellular carbon flux.

In many organisms, $\mathrm{NAD}(\mathrm{P})^{+}$-dependent transhydrogenases play an important role in maintaining cellular redox poise and regenerating $N A D^{+}$and $\mathrm{NADPH}$ by catalyzing the reversible reaction: $\mathrm{NADH}+\mathrm{NADP}^{+} \rightleftarrows$ $N A D^{+}+\mathrm{NADPH}[6]$. Yeasts, however, do not encode $\mathrm{NAD}(\mathrm{P})^{+}$-dependent transhydrogenases [7] and therefore native yeast strains must rely on other methods to regenerate redox potential. Heterologous expression of membrane-bound and soluble transhydrogenases from Azotobacter vinelandii and Escherichia coli in Saccharomyces cerevisiae have been unsuccessful in generating NADPH, instead catalyzing the reverse reaction resulting in increased levels of NADH when cultured in glucose [8-10]. Therefore, expression of bacterial transhydrogenases is thought not to be a viable route for NADPH production in S. cerevisiae.

As an alternative, many oleaginous yeasts encode a cytosolic malic enzyme and are poised to utilize a three-enzyme transhydrogenase-like cycle often referred to as the pyruvate-oxaloacetate-malate or "POM" cycle [11]. In the POM cycle, pyruvate is converted to oxaloacetate through the activity of pyruvate carboxylase (Pyc), oxaloacetate is subsequently converted to malate by malate dehydrogenase (Mdh), and then malate is converted back to pyruvate in the cytosol through the activity of Malic enzyme (Mae) (Figure 1). The net stoichiometry of these three enzymatic reactions is ATP $+\mathrm{NADH}+\mathrm{NADP}$ à $A D P+\mathrm{P}_{\mathrm{i}}+$ $\mathrm{NAD}^{+}+\mathrm{NADPH}$ and is essentially identical to the net stoichiometry of the bacterial transhydrogenase except that ATP is also utilized [6]. The presence of a cytosolic malic enzyme is important in these shunts as NADPH cannot cross the mitochondrial membrane and cells maintain distinct cytosolic and mitochondrial pools of NADPH. Also salient is the fact that the cycle regenerates pyruvate, a key intermediate in many cellular processes and does not alter the net carbon flux. The lack of a cytosolic malic enzyme in S. cerevisiae and in the emergent model oleaginous yeast, Yarrowia lipolytica means that a fully cytosolic version of the POM cycle does not naturally exist in these yeasts. Instead much of 
the cytosolic reducing power needed for anabolic reactions is provided through the glucose-6-phosphate dehydrogenase (Zwf1) and 6-phosphogluconate dehydrogenase (Gnd1) steps of the pentose phosphate pathway, the cytosolic NADP ${ }^{+}$-specific isocitrate dehydrogenase (Idp2) and the NADP ${ }^{+}$-dependent aldehyde dehydrogenase (Ald6) [12]. Mitochondrial NADPH is generated through the mitochondrial NADH kinase (Pos5), through the activity of aldehyde dehydrogenases (Ald4/5) and through the activity of mitochondrial NADP ${ }^{+}$-dependant malic enzymes (Mae1) [13].

The overexpression of endogenous enzymes to create synthetic POM cycles has been employed in $S$. cerevisiae to counter redox imbalance imposed through the introduction of synthetic xylose metabolism, isobutanol production pathways and to increase the pools of NADPH in different cellular compartments $[12,14-16]$. These POM cycles have overexpressed the endogenous protein isoforms Pyc2 and Mdh2 and either the endogenous malic enzymes (Mae1) or a truncated cytosolic version (sMae1). More recently, a POM cycle was establishing utilizing Pyc1, Mdh3 with a deletion of its peroxisome targeting signal ('Mdh3) and a heterologous malic enzyme from the oleaginous yeast Rhodosporidium toruloides (RtME) as part of an engineering effort to reprogram $S$. cerevisiae from alcoholic fermentation to lipogenesis [17]. However, a comparison of the efficacy of the various shunts has not been reported.

cerevisiae encodes three isoforms of malate dehydrogenase each with distinct cellular functions that may affect their efficacy in a transhydrogenase-like shunts. Mdh1 is a mitochondrial enzyme that operates in the TCA cycle and as part of the malate/aspartate shunt but functions primarily to synthesize oxaloacetate from malate $[18,19]$. Mdh2 is cytosolic and functions primarily in gluconeogenesis to make malate from oxaloacetate, but is recognized by ubiquitin ligase and targeted for proteolysis in glucose containing media [20-23]. Finally, Mdh3 is peroxisomal and functions in the glyoxylate cycle [24]. $S$. cerevisiae also encodes two isoforms of pyruvate carboxylase, Pyc1 and Pyc2. The distinct roles of the cytosolic isoforms of pyruvate carboxylase in S. cerevisiae are less clear, however the two isoforms Pyc1 and Pyc2 appear to be differently regulated in response to carbon and nitrogen sources, with Pyc1 being reported to have higher activity in glucose [25-27]. The isoforms of these enzymes differ in their subcellular localization, protein stabilities and cellular functions in ways that may influence the effectiveness of any synthetic POM cycle into which they are incorporated. The effectiveness of different combinations of Mdh and Pyc isoforms as part of synthetic POM cycles has not been reported.

The final method of transforming NAD to NADP - the method that is employed in native strains of $S$. cerevisiae - is through the activity of NAD kinases [28]. NAD kinases utilize ATP to catalyze the phosphorylation of $\mathrm{NAD}^{+}$or NADH. S. cerevisiae encodes three NAD kinase isoforms, YEF1, UTR1 and POS5 [29]. POS5 encodes a mitochondrial NADH kinase that has lower activity towards NAD', preferentially phosphorylating NADH to generate NADPH. In S. cerevisiae the mitochondrial NADH kinase, Pos 5 is a significant source of mitochondrial NADPH [13] and both the endogenous Pos 5 and a modified isoform lacking the mitochondrial signal sequence have been used successfully to counter redox imbalance in metabolic engineering studies [30-32]. YEF1 and UTR1 encode paralogs that are both located in the cytosol and display activity towards both, NAD ${ }^{+}$and NADH. Overexpression of UTR1 has 
been shown to improve ethanol production in an engineered strain of S. cerevisiae [33], however, to the best of our knowledge overexpression of YEF1 has not been investigated for its effects on biosynthetic processes and a general comparison of the effectiveness of overexpression of these NAD kinases on biosynthetic processes is lacking.

The aim of this study is to compare the efficacy of these general methods for converting the abundant $\left(\mathrm{NAD}^{+}\right.$and NADH) redox cofactors into NADPH for fatty acid-based chemical production in S. cerevisiae. We established and tested four synthetic POM cycles composed of distinct combinations of malic enzyme, pyruvate carboxylase and malate dehydrogenase isoforms for their effects on fatty alcohol production. In addition, we investigated the effects of cytosolic overexpression of the three endogenous NAD kinases on fatty alcohol production. Finally, we investigated whether the effects of overexpression of the best performing POM cycle, containing PYC1, 'MDH2 and sMAE1 could have additive effects when combined with the overexpression of NAD kinases on fatty alcohol production in S. cerevisiae.

\section{Methods}

\section{Strains, media and cultivations}

coli DH5a was used for the construction and propagation of all plasmid constructs. E. colistrains were cultured at $37^{\circ} \mathrm{C}$ in lysogeny broth (LB) supplemented with ampicillin $(100 \mu \mathrm{g} / \mathrm{mL})$. S. cerevisiae strains were grown in either YPD medium (Yeast extract $10 \mathrm{~g} / \mathrm{L}$, peptone $20 \mathrm{~g} / \mathrm{L}, \mathrm{D}-\mathrm{glucose} 20 \mathrm{~g} / \mathrm{L}$ ) or synthetic media lacking leucine (-leu), uracil (-ura), or both leucine and uracil (-leu -ura) as appropriate $(20 \mathrm{~g} / \mathrm{L}$ glucose, $5 \mathrm{~g} / \mathrm{L}$ ammonium sulfate, $1.7 \mathrm{~g} / \mathrm{L}$ yeast nitrogenous base (YNB) without amino acids and $2 \mathrm{~g} / \mathrm{L}$ of amino acid drop out mix). S. cerevisiae strains were cultured in $25 \mathrm{~mL}$ of medium in $250 \mathrm{~mL}$ Erlenmeyer flasks at $30^{\circ} \mathrm{C}$ at $200 \mathrm{rpm}$ for 72 hours with a $10 \% \mathrm{v} / \mathrm{v}$ dodecane overlay.

\section{Plasmid and strain construction}

Plasmids and strains used in this study are listed in Table 1; oligonucleotides utilized for plasmid and strain construction are listed in Table S1. The PYC1, PYC2, MDH1, and $M D H 2$ open reading frames and transcriptional terminators, the open reading frame of MAE1, and the TEF1, HXT7 and FBA1 promoters were amplified from $S$. cerevisiae W303 genomic DNA using the appropriate oligonucleotide primers (Table S1). The oligonucleotides were designed to delete amino-terminal coding sequence implicated in protein stability $(M D H 2)$ or targeting the enzyme to the mitochondria (MDH1, MAE1) and to introduce necessary homologies and restriction sites for assembling the genes into distinct combinations for POM cycle expression (Fig. 1B). Briefly, the yeast/E. coli shuttle vector, YEplac181 [34] was digested with BamHI and Smal and the HXT7 promoter, the FBA1 promoter and truncated SMAE1 gene were inserted via Gibson isothermal assembly [35]. The resultant vector was digested with Smal and Avrll and an additional round of isothermal assembly reactions, inserting the TEF1 promoter, and either isoform PYC1 or $P Y C 2$, and $M D H 1$ or $M D H 2$, was performed to create four unique POM cycle expressing cassettes (Table 1; Figure S1). To reduce the variability in expression associated with use of high-copy episomal 
plasmids the gene cassettes were transferred to the centromere containing plasmid YCplac111 as diagrammed in Figure S2. Sequences corresponding to the carboxyl-terminal region of MAE1 and the transcriptional terminator of $C D C 28$ were PCR amplified (Table S1). The fragments were then combined via overlap extension PCR. The resultant PCR product was inserted into the Sacl - EcoRI digested YCplac111 to create the intermediate vector YCplac111-fMAE1-CDC28t (Figure S2). Subsequently, each of the vectors YEplac181-NC1/2/3/4 were digested with Sacl and Sall, and the resultant fragment was ligated with Sacl - Sall digested YCplac111-fMAE1-CDC28t to generate the YCpNC1/2/3/4 vectors (Figure S2; Table 1). A control plasmid expressing MAE1 under control of the FBA1 promoter was constructed by amplifying the PFBA1-sMAE1 fragment with the oligonucleotides Sall-FBA1p and MAEC3. The resulting fragment was digested with Sall and Sacl and inserted into the intermediate vector YCplac111-fMAE1-CDC28t. The other single gene control vectors for the other constructs were generated both with and without a carboxyl-terminal MYC epitope tag via Gibson assembly utilizing oligos listed in Table S1. Briefly, promoter, gene, and terminator were amplified from the YCpNC1-4 plasmids either as a single fragment (untagged) or as two fragments to insert the MYC epitope tag and assembled into YCplac111 linearized with BamHI and Smal.

The YEplac195bb-PPGK1-ScFAR plasmids were constructed by inserting a codon optimized version of Mus musculus FAR1 (ScFAR) digested with Xbal and Pstl into YEpLac195-PPGK1 digested with Spel and Pstl [36]. Genes encoding S. cerevisiae NAD kinases UTR1, YEF1, and POS5c were amplified from genomic DNA, digested by Xbal and Pstl, except POS5c which was digested with Avrll and Pstl, and subsequently ligated into YEpLac195-PPYK1 linearized with Spel and Pstl. The PPGK1-SCFAR fragment was excised from its vector by digesting with $\mathrm{Xbal}$ and Pstl and the PPGK1-SCFAR1 fragment was subsequently ligated into Spel - Pstl cut vectors harbouring each of the NAD kinase open reading frames.

For overexpression of FAS1 a 3430bp DNA fragment encompassing the 5 ' coding sequence of FAS1 was ligated behind a $P G K 1$ promoter fragment (PPGK1) in integrating vector pRS303. The vector was digested with $\mathrm{Pacl}$ to target integration to FAS1 thus placing the chromosomal FAS1 coding sequence under control of the PGK1 promoter.

All plasmids were verified by sequencing. All constructs were introduced into $S$. cerevisiae by lithium acetate mediated transformation [37] selecting for transformants on the appropriate synthetic medium. All resulting strains were verified by PCR amplification of diagnostic fragments from total chromosomal DNA.

\section{Protein extraction and detection via western blots}

Control strain BMY01 and strains BMY07-BMY10 expressing MYC tagged proteins were inoculated and cultured overnight in YPD medium and then were diluted 1 in 25 in fresh YPD and incubated at $30^{\circ} \mathrm{C}$ until an $\mathrm{OD}_{600}$ of 0.5 was achieved. Total protein was extracted from $10 \mathrm{~mL}$ of cell culture via a trichloroacetic acid (TCA) extraction protocol [38]. 
Protein samples were separated by electrophoresis using $8 \%$ SDS-polyacrylamide gels and transferred to a PVDF membranes. The membranes were subsequently incubated with anti-MYC mouse monoclonal antibody $9 \mathrm{E} 10(1: 10,000)$ followed by washing and incubation with HRP-conjugated goat anti-mouse IgG prior to visualization of the protein species with enhanced chemiluminescence. The membranes were stripped and probed with mouse monoclonal anti-PSTAIRE to detect Cdc28 as a loading control.

\section{RNA extraction and RT-qPCR analysis}

RNA was prepared according to the protocol outlined in by Schmitt ME, Brown TA and Trumpower BL [39]. Briefly, cell pellets from $1 \mathrm{~mL}$ of culture were frozen at $-70^{\circ} \mathrm{C}$. These were subsequently thawed on ice, resuspended in $400 \mu \mathrm{L}$ AE Buffer (50 mM NaOAc pH 5.3, 10 mM EDTA - RNA grade) and transferred to RNase free microcentrifuge tubes. $40 \mu \mathrm{L}$ of $10 \%$ SDS was added to each sample followed by $500 \mu \mathrm{L}$ prewarmed AE-phenol and mixed by inverting. Samples were incubated at $65^{\circ} \mathrm{C}$ for 5 minutes, mixing occasionally. Samples were rapidly chilled in a dry ice ethanol bath for approximately 5 seconds and then centrifuged to allow for separation into organic and aqueous phases. Aqueous phases were recovered, extracted a second time with an equal volume of acid phenol chloroform. Nucleic acids in the aqueous fraction were precipitated by the addition of $40 \mu \mathrm{L} 3 \mathrm{M} \mathrm{NaOAc} \mathrm{pH} 5.3$ (RNA grade) and 2.5 volumes of $100 \% \mathrm{EtOH}$ (RNA grade) on dry ice. Samples were pelleted, ethanol removed and air dried prior to resuspension in $30 \mu \mathrm{L}$ DEPC-treated $\mathrm{H}_{2} 0.5 \mu \mathrm{L}$ of each RNA prep were separated on a 1\% bleach gel [40] for assessment of RNA quality. Subsequently the purity and concentration of the RNA samples were determined via nanodrop. Nucleic acid preparations were digested with RNase-free DNase I at room temperature for 15 minutes. Digestion were stopped by the addition of $1 \mu \mathrm{L} 25 \mathrm{mM}$ EDTA and heating to $65^{\circ} \mathrm{C}$ for 10 minutes. cDNA synthesis was performed from each RNA sample, using $1 \mu \mathrm{g}$ of total RNA and the iScript reverse transcriptase supermix for RT-qPCR (Biorad) according to manufacturer's protocol. No-RT controls were performed with the omission of the RT. qPCR reactions were in a total volume of 10 $\mu \mathrm{l}$ containing $10 \mathrm{pmol}$ of forward and reverse primers (as listed in Table S2), $5.0 \mu \mathrm{L}$ 2X iQ SYBR Green Supermix (Bio-Rad) and $1.25 \mu \mathrm{L}$ of the previous RT reaction as template. A three-cycle amplification was performed $\left(95^{\circ} \mathrm{C}\right.$ for $10 \mathrm{~s}, 54^{\circ} \mathrm{C}$ for $15 \mathrm{~s}, 72^{\circ} \mathrm{C}$ for $20 \mathrm{~s}$ ) with melt curve (ramping from $72^{\circ} \mathrm{C}$ to $95^{\circ} \mathrm{C}$ ) using the Rotor-Gene Q, real-time PCR cycler (Qiagen). No-RT and no-template controls were included in each run, and all unknowns were run in technical triplicates. Actin specific primers were used to normalize the data. Amplification efficiency of all primer pairs were tested prior to use in RT-qPCR and were found to be between $90-105 \%$ efficiency.

\section{Fatty alcohol extraction and analysis}

Cell cultures $(25 \mathrm{~mL})$ were overlaid with dodecane $(2.5 \mathrm{~mL})$ spiked with a pentadecanol $(\mathrm{C} 15: 0 \mathrm{OH})$ internal standard. Following the 72-hour growth period, the culture was pelleted by centrifugation ( $2800 \mathrm{x}$ $\mathrm{g}, 5 \mathrm{~min}, 4^{\circ} \mathrm{C}$ ). The dodecane layer was isolated and then either used directly or $100 \mu \mathrm{L}$ of the upper dodecane phase was diluted in $900 \mu \mathrm{L}$ ethyl acetate. A $1 \mu \mathrm{L}$ aliquot was analyzed via GC-FID using an Agilent 6890 series GC System equipped with a CP-Wax 58 FFAP CB column $(25 \mathrm{~m} \times 320 \mu \mathrm{m} \times 0.2 \mu \mathrm{m}$, Agilent, USA). Helium was used as a carrier gas, with a constant flow of $1 \mathrm{~mL} / \mathrm{min}$. The temperature of 
the inlet was $240^{\circ} \mathrm{C}$. The following program was applied: $50^{\circ} \mathrm{C}$ for 1 minute, increase $25^{\circ} \mathrm{C} /$ minute to $170^{\circ} \mathrm{C}$ followed by $10^{\circ} \mathrm{C} / \mathrm{min}$ to $240^{\circ} \mathrm{C}$, hold at $240^{\circ} \mathrm{C}$ for 7 minutes. The internal standards were used to determine product yields, in conjunction with calibration curves and known relative response factors. Mean values were determined from three independent biological replicates unless otherwise stated.

Table 1. Plasmids and strains used in this study 
YEplac181

YEplac181-

PHXT7*-PFBA1-

MAE1

YEpNC1

YEpNC2

YEpNC3

YEpNC4

YCplac111

YCplac111-

fMAE1-CDC28t

YCpNC1

YCpNC2

YCpNC3

YCpNC4

YCPMAE1

YCpPYC1

YCpPYC2

YCp'MDH1

YCp'MDH2

YCpPYC1-myc

YCpPYC2-myc

YCp'MDH1-myc

YCp'MDH2-myc

YEpScFAR
AmpR; High copy LEU2 S. cerevisiae - E. coli shuttle vector

[34]

AmpR; YEplac181 derivative containing the S. cerevisiae HXT7 and $F B A 1$ promoters separated by Avrll and Smal restriction sites and $S M A E 1$

AmpR; YEplac181 derivative containing PHXT7-PYC1 PTEF1-'MDH2 - PFBA1-sMAE1

AmpR; YEplac181 derivative containing PHXT7-PYC1 PTEF1-'MDH1 - PFBA7-sMAE1

AmpR; YEplac181 derivative containing PHXT7-PYC2 -

PTEF1-'MDH2 - PFBA1-SMAE1

This work

AmpR; YEplac181 derivative containing PHXT7-PYC2 -

PTEF1-'MDH1 - PFBA1-sMAE1

This work

[34]

AmpR; CEN plasmid LEU2 S. cerevisiae - E. coli shuttle vector

This work AmpR; YCplac111 derivative con
the $C D C 28$ terminator sequence

AmpR; YCplac111 derivative containing PHXT7-PYC1 PTEF1-'MDH2 - PFBA1-sMAE1

This work

AmpR; YCplac111 derivative containing PHXT7-PYC1 -

PTEF1-'MDH1 - PFBA1-sMAE1

This work

AmpR; YCplac111 derivative containing PHXT7-PYC2 -

PTEF1-'MDH2 - PFBA1-sMAE1

This work

AmpR; YCplac111 derivative containing PHXT7-PYC2 -

PTEF1-'MDH1 - PFBA1-SMAE1

This work

AmpR; YCplac111 derivative containing PFBA1-sMAE1

This work

AmpR; YCplac111 derivative containing PHXT7-PYC1

This work

AmpR; YCplac111 derivative containing PHXT7-PYC2

This work

AmpR; YCplac111 derivative containing PTEF1-'MDH1

This work

AmpR; YCplac111 derivative containing P TEF1-'MDH2

This work

AmpR; YCplac111 derivative containing PHXT7-PYC1-myC

This work

AmpR; YCplac111 derivative PHXT7-PYC2-myc

This work

AmpR; YCplac111 derivative containing PTEF1-'MDH1-myc

This work

AmpR; YCplac111 derivative containing PTEF1-'MDH2-myc

This work

AmpR; YEplac195 derivative containing pPGK1 driving expression of the Mus musculus FAR1 gene codon optimized for
This work 
expression in S. cerevisiae

YEpPOS5c/ScFAR AmpR; YEplac195 derivative containing PPYK1 driving

expression of a cytosolic version of Pos5 and PPGK1-SCFAR

This work

YEpUTR1/ScFAR

AmpR; YEplac195 derivative containing PPYK1 driving

This work

expression of UTR1 and PPGK1-SCFAR

YEPYEF1/ScFAR

AmpR; YEplac195 derivative containing PPYK1 driving

This work expression of YEF1 and PPGK1-SCFAR

pRS303-PGK1- AmpR; $p R S 303$ derivative containing PPGK1 driving expression FAS1 of FAS1

Courtesy of

XiaoDong

Liu

Strains

S. cerevisiae

W303

BMY01

BMY02

BMY03

BMY04

BMY05

BMY06

BMY07

BMY08

BMY09

BMY10

FAS1

BMY11

BMY12

BMY13

BMY14

BMY15

BMY16

BMY17

BMY18

\section{Relevant Genotype}

MATa leu2-3,112 trp1-1 can1-100 ura3-1 ade2-1 his3-11,15

W303 YCplac111

W303 YCpNC1

W303 YCpNC2

W303 YCpNC3

W303 YCpNC4

W303 YCpMAE1

W303 + YCp'MDH1-myc

W303 + YCp'MDH2-myc

W303 + YCpPYC1-myc

W303 + YCpPYC2-myc

W303 fas 1::PPGK1-FAS1-HIS3

FAS1 YEpScFAR

FAS1 YCplac111 YEpScFAR

FAS1 YCpNC1 YEpScFAR

FAS1 YCpNC2 YEpScFAR

FAS1 YCpNC3 YEpScFAR

FAS1 YCpNC4 YEpScFAR

FAS1 YCpMAE1 YEpScFAR

FAS1 YCp'MDH1 YEpScFAR
Source

Open

Biosystems

This work

This work

This work

This work

This work

This work

This work

This work

This work

This work

This work

This work

This work

This work

This work

This work

This work

This work

This work 
BMY20

FAS1 YCpPYC1 YEpScFAR

This work

BMY21

FAS1 YCpPYC2 YEpScFAR

This work

MATa his3D1 leu2D0 met15D0 ura3D0 zwf1::KanMX4

Open

Biosystems

BY4741 zwf1

BMY22

BY4741 zwf1 YCplac111 YEpScFAR

This work

BMY23

BY4741 zwf1 YCpNC1 YEpScFAR

This work

BMY24

BY4741 zwf1 YCpNC2 YEpScFAR

This work

BMY25

BY4741 zwf1 1 YCpNC3 YEpScFAR

This work

BMY26

BY4741 zwf1 Y YCpNC4 YEpScFAR

This work

BMY27

FAS1 YCplac111 YEpUTR1/ScFAR

This work

BMY28

FAS1 YCplac111 YEpYEF1/ScFAR

This work

BMY29

FAS1 YCplac111 YEpPoS5c/ScFAR

This work

BMY30

FAS1 YCpNC1 YEpUTR1/ScFAR

This work

BMY31

FAS1 YCpNC1 YEpYEF1/ScFAR

This work

BMY32

FAS1 YCpNC1 YEpPoS5c/ScFAR

This work

\section{Results And Discussion}

\section{Construction of Synthetic POM Cycles}

Gene cassettes encoding four distinct POM cycles were constructed in the Yeast/ E.coli shuttle vector YCplac111 (YCpNC1-4; Table 1). Each cassette contains a truncated version of the S. cerevisiae malic enzyme gene $M A E 1$, designated $S M A E 1$, lacking the first 90 nucleotides which encode amino acids believed to effect targeting to the mitochondria [41]. Lacking this signaling sequence it is expected that sMae1 will localize to the cytosol. The cassettes also contain the gene sequences for either of the endogenous S. cerevisiae isoforms of pyruvate carboxylase (PYC1 or PYC2) and an isoform of malate dehydrogenase ('MDH1 or 'MDH2) resulting in four combinatorial arrangements (Figure 1B).

Differing from most other eukaryotic cells, $S$. cerevisiae encodes two isoenzymes of pyruvate carboxylase that are localized to the cytosol rather than a single mitochondrial enzyme. Expression of the isoforms is differentially regulated at the level of transcription depending on factors such as growth phase, carbon source and nitrogen source [25-27], however, in the gene cassettes the coding sequences were placed under the regulation of an HXT7 promoter to eliminate differential transcriptional regulation. 
However, the protein isoforms display different allosteric properties with respect to acetyl-CoA activation and aspartate inhibition [42] with Pyc1 displaying a higher degree of cooperativity. In addition, Pyc2 reportedly displays lower abundance and reduced activity in glucose [25]. Therefore, it is reasonable to predict that these isoforms may display distinct differences when incorporated into the synthetic POM cycles.

For the expression of malate dehydrogenase, we aimed to utilize versions that would be both cytosolic and stable in medium containing glucose as the carbon source. To achieve this, a nucleotide sequence corresponding to the 17 amino acid mitochondrial signal sequence from $M D H 1$ [19] was deleted in order to avoid targeting to the mitochondria. Mdh2 is subject to both transcriptional repression and phosphorylation-mediated post-translational degradation in the presence of glucose [21]; therefore, to stabilize Mdh2, the nucleotide sequence encoding 12 amino acids at the amino-terminus was deleted in the overexpression constructs used in this study. Control plasmids consisting of each of the genes individually expressed were also generated. The proposed mechanism of carbon shuttling and generation of NADPH by these shunts is shown in Figure 1 along with the normal localization and proposed altered localization of the overexpressed forms.

\section{Verification of protein isoform expression}

cerevisiae strains harboring vectors for expression of the individual $P Y C 1, P Y C 2, M D H 1$ and $M D H 2$ variants were assayed by western blot to confirm accumulation of the respective protein products. Pyc1 and Pyc2 were both expressed in the test strains but Pyc1 accumulated to a higher level than Pyc2 (Fig 2). The open reading frames of $P Y C 1$ and $P Y C 2$ were both under the regulation of an $H X T 7$ promoter, which was expected to limit the differences in transcriptional regulation between the two but qPCR analysis demonstrated that the mRNA for PYC2 accumulated to higher levels than the mRNA for PYC1 (Fig. 3B). Thus, these results indicate that either Pyc1 is translated more efficiently or is more stable than Pyc2. This is similar to the distinctly higher abundance and activity of the endogenous Pyc1 when cells are cultured in medium with glucose as carbon source [25]. In the context of utilizing a synthetic POM cycle to generate $\mathrm{NADPH}$, the stoichiometry of the enzyme activities is likely to be as important as the total activity for optimal function. In this case, high pyruvate carboxylase activity may not be optimal for operation of the POM cycle unless it can be matched by the activity of the malate dehydrogenase and malic enzyme. The Mdh1 and Mdh2 variants we tested displayed similar abundance to one another supporting the contention that the amino-terminal truncation of Mdh2 would stabilize the enzyme when cells were fermenting glucose as a carbon source. It is also worth noting that both malate dehydrogenase variants display steady state abundance similar to Pyc1 (Fig 2). Interestingly, when mRNA was monitored in strains expressing the full POM cycle vectors, we observed that strains overexpressing 'MDH1 saw a concurrent increase in mRNA from the endogenous $M D H 2$ while this did not appear to be true for cells overexpressing ' $\mathrm{MDH} 2$ (Fig. 3A). There is no published report of Mdh1 influencing the expression of $M D H 2$ but it is unlikely that this would be detrimental to the functioning of the synthetic POM cycle. The sMAE1 allele was constant between the four POM cycles and levels of the expression of this enzyme were not evaluated. 


\section{Effect of Synthetic POM cycles on Fatty Alcohol Production}

As increasing NADPH production is extremely salient for the production of oleochemicals in microbial systems, we investigated the effects of the four synthetic POM cycles in strains minimally engineered towards fatty alcohol production. These strains (BMY12 - BMY16; Table 1) overexpress FAS1 under regulation of the strong constitutive $P G K 1$ promoter (PPGK1), express a codon-optimized version of the Mus musculus fatty acyl-CoA reductase (ScFAR) and express a synthetic POM cycle. Increased expression of $F A S 1$ results in a coinciding increase in the levels of $F A S 2$ yielding an overall increase in the expression of the FAS complex [43]. This increases fatty acid biosynthesis activity, ultimately creating an increased demand for NADPH in the cells. As the reactions catalyzed by both the FAS complex and ScFAR1 enzyme are NADPH-dependent (Figure 1) we hypothesized the availability of NADPH may become limiting in this synthetic pathway and that expression of the synthetic POM cycles would increase availability of NADPH and drive increased flux through the pathway resulting in an increased titer of fatty alcohols.

Seven independent colonies from each transformation (BMY12 - BMY17) were tested for fatty alcohol production in shake flask culture. There was a high degree of variability in the titer of fatty alcohols produced among individual transformants carrying the same plasmid, particularly in those strains that produced the highest levels of fatty alcohols (Figure S3). Upon further investigation, we found that the variation among transformants harbouring the same plasmid was largely accounted for by some colonies being high producers while others were low producers (Fig S3). The production characteristic of a transformant was clonal. When a high producing colony was streaked to isolate independent colonies all of those plasmid-bearing colonies consistently maintain high levels of production. Three single colonies were isolated from each of the highest producing candidates from the strains (BMY12 - BMY17) and tested for fatty alcohol production (Figure 3A). The BMY13 strain ( $P Y C 1,{ }^{\prime} M D H 2$, sMAE1) displayed an increase in fatty alcohol production relative to the other synthetic POM cycles and the controls. Specifically, BMY13 increased fatty alcohol production by 40\% from the control strain BMY12. resulting in production titers of $68.6 \pm 3.3 \mathrm{mg} / \mathrm{L}$ and $49.0 \pm 2.2 \mathrm{mg} / \mathrm{L}$ respectively. Interestingly, an $\sim 20 \%$ decrease in fatty alcohol production was observed for the PYC2 overexpressing strains BMY15 and BMY16, producing $36.8 \pm 1.2 \mathrm{mg} / \mathrm{L}$ and $38.3 \pm 3.7 \mathrm{mg} / \mathrm{L}$ respectively, however this decrease in productivity can be accounted for by the lower cell density. When normalized for culture density BMY15 (PYC2, 'MDH2, SMAE1) and BMY16 (PYC2, 'MDH1, sMAE1) produced fatty alcohol similar to the vector control strain (Figure S4). Overexpression of SMAE1, or either isoform of PYC or MDH alone (BMY17 - BMY21) showed no increase in fatty alcohol production relative to the empty vector control strain (BMY12; Figure S5).

Although the YCpNC1 plasmid (PYC1, 'MDH2, sMAE1) in strain BMY13 was the only synthetic POM cycle to result in increased fatty alcohol production in our FAS overexpressing background, all combinations of the POM cycle enzymes should be capable of increasing NADPH availability to support elevated fatty alcohol production and so we opted to test the effects of the synthetic POM cycles in a second strain background. We went on to investigate whether the synthetic POM cycles would be capable of increasing fatty alcohol production in a zwf1D strain expressing ScFAR (BMY22 - BMY26). As the zwf1D strain 
cannot generate NADPH through the pentose phosphate pathway (Figure 1) - one of the primary sources of NADPH production in actively proliferating $S$. cerevisiae -it is expected that fatty alcohol production in these strains may be improved by alternate sources of NADPH production, such as the synthetic POM cycles. Again, we observed that strains producing higher titers of fatty alcohols had greater variability in production between individual colony replicates (Figure 4, BMY22-BMY24 vs BMY25 and BMY26). Strains containing the plasmids YCpNC1 (PYC1, 'MDH2, sMAE1) and YCpNC2 (PYC1, 'MDH1, sMAE1) (BMY23 and BMY24, respectively; Table 1) displayed increased fatty alcohol production when compared to the strains containing YCpNC3 (PYC2, 'MDH2, sMAE1) and YCpNC4 (PYC1, 'MDH1, sMAE1), BMY25 and BMY26 produced lower levels of fatty alcohols (Figure 4). Production of fatty alcohols by BMY24, however, was comparable to the control strain BMY22. This trend remained true when the effects of biomass accumulation were also taken into account and fatty alcohol production was expressed as $\mathrm{mg} / \mathrm{L} / \mathrm{OD}$ (Figure S6).

Although all combinations of the POM cycle enzymes should be capable of increasing NADPH availability resulting in elevated fatty alcohol production, we found that only YCpNC1 (PYC1, $M D H 2$, SMAE1) had appreciable effects on fatty alcohol production in both the FAS overexpressing and $z w f 1 \Delta$ strains. As such, it may be that some combinations of POM cycle enzymes lead to unexpected metabolic consequences or increased metabolic burden with little commensurate benefit to redox cofactor biosynthesis. This appears to be especially true of strains overexpressing PYC2 (BMY15, BMY16, BMY25 and BMY 26; Figures 3 and 4), where both reduced cell growth (Figure 3 ) and decreased fatty alcohol production were observed (Figures 3 and 4). Due to this, we conclude that strains overexpressing PYC1 as part of a synthetic POM cycle outperformed those overexpressing PYC2. Why Pyc1 out performs Pyc2 in these cycles is not clear. It may simply be that Pyc1 accumulates to higher levels than Pyc2 (Figure 2) or that the relative abundance of Pyc1 and the Mdh expressed results in more stoichiometric expression. However, we cannot discount the possibility that the functional differences between the isoforms in this context is due to allosteric regulation by cellular metabolites or other inherent differences between to two protein isoforms.

The POM cycle employing Pyc1 Mdh2 sMae1 yielded higher fatty alcohol production than did the Pyc1 Mdh1, sMae1 cycle (Figures 3 and 4). This demonstrates that Mdh2 is more effective in the context of this cycle. The coding sequences for the two isoforms were modified to provide stable cytosolic expression in glucose and showed comparable protein abundance in actively proliferating cells (Figure 2); however, Mdh2 is native to the cytosol whereas Mdh1 normally functions within the environment of the mitochondria with a different redox environment. It may also be significant that Mdh2 displays a higher affinity for NADH that does Mdh1, which may make it more effective in driving the oxaloacetate to malate reaction [24]. Another isoform of malate dehydrogenase, the peroxisome associated Mdh3 has also been employed in a POM cycle [17]. However, Mdh3 has a significantly lower affinity for oxaloacetate than

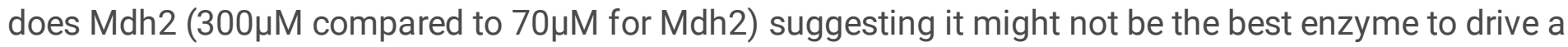
POM cycle for NADPH synthesis. 
The early reports on application of synthetic POM cycles for metabolic engineering in S. cerevisiae all employ the endogenous Pyc2 isoform, along with Mdh2 and Mae1. They have been reported to counter the redox imbalance of a heterologous xylose fermentation pathway [16] and for the production of isobutanol in S. cerevisiae [15]. However, while the expression of the cycle in xylose fermenting conditions was reported to lead to an increased rate of xylose consumption, ultimately it resulted in decreased ethanol production [16]. When implemented in isobutanol producing strains, a version of the cycle expressing a truncated Mae1 (sMae1p) expected to locate to the cytosol, was found to increase isobutanol production in a strain that overexpressed the Ehrlich pathway and ILV2 but was found to have no effect on isobutanol titers in other strains [15]. While the efficacy of these POM cycles is not immediately clear $[12,14-16]$, it is possible that they may be improved by overexpressing Pyc1 rather than Pyc2 as we have shown that enzyme combinations including Pyc1, particularly when combined with Mdh2, out perform those containing Pyc2. Adding support to this argument, a recent report converting $S$. cerevisiae to lipogenic metabolism utilized a Pyc1 containing POM cycle with success, however alternate combinations were not reported [17].

\section{Effect of NAD Kinase expression on fatty alcohol production}

An alternative to the use of synthetic POM cycles to increase NADPH, is to express enzymes with NAD kinase activity. To determine whether overexpression of NAD kinase activity could improve fatty alcohol biosynthesis, YEF1, UTR1 and a cytosolic version of POS5, denoted POS5C were introduced into a highcopy plasmid harbouring ScFAR1 and these constructs were installed in S. cerevisiae. Overexpression of UTR1 (BMY27) resulted in a slight increase in fatty alcohol production over the control strain BMY12, increasing from 82 to $90 \mathrm{mg} / \mathrm{L}$ (Figure S7) or $7.23 \mathrm{mg} / \mathrm{L} / \mathrm{OD}$ to $8.13 \mathrm{mg} / \mathrm{L} / \mathrm{OD}$ (Figure 5). Overexpression of cytosolic POS5C (BMY29) resulted in a significant increase in fatty alcohol titers from $82 \mathrm{mg} / \mathrm{L}$ to 111 $\mathrm{mg} / \mathrm{L}$ or $7.23 \mathrm{mg} / \mathrm{L} / \mathrm{OD}$ to $11.95 \mathrm{mg} / \mathrm{L} / \mathrm{OD}$ (Figures S7 and Figure 5). Overexpression of YEF1, on the other hand, failed to improve titers. On their own we found that only Pos $5 \mathrm{c}$ was truly effective at increasing fatty alcohol production and that the increase in production was comparable to expressing the best POM cycle (PYC1, 'MDH2, sMAE1). In this independent trial, we found that expressing YCpNC1 in strain BMY13, produced $119 \mathrm{mg} / \mathrm{L}$ fatty alcohols while expressing Pos5c in strain BMY29 resulted in 111 $\mathrm{mg} / \mathrm{L}$ fatty alcohols. Compared to the vector control strain BMY12 $\left(\mathrm{OD}_{600}=11.5\right)$, both BMY29 and BMY13 displayed a slight decrease in biomass accumulation $\mathrm{OD}_{600}=9.3,10.04$ respectively (Figure S7A). The fact that Pos5c was the only NAD kinase to show a significant increase in fatty alcohol production is likely due to the fact that mitochondrial Pos5 is a NADH kinase that phosphorylates NADH preferentially with much lower activity towards $\mathrm{NAD}^{+}$. Yef 1 and Utr1 are paralogs that both are located in the cytosol and display activity towards both $\mathrm{NAD}^{+}$and $\mathrm{NADH}$ substrates.

We next sought to investigate whether expression of the NAD kinases and our synthetic POM cycle could be combined to further increase fatty alcohol production. When combined with YCpNC1 encoding POM cycle enzymes PYC1, 'MDH2, and SMAE1, the overexpression of YEF1 and UTR1 had similar effects as they did when expressed with the empty vector control YCplac111 (Figure 5). UTR1 overexpression combined with YCpNC1 (BMY30) resulted in a slight increase in fatty alcohol production compared to the 
control expressing YCpNC1 (BMY13) (125 mg/L vs. $119 \mathrm{mg} / \mathrm{L}$ or $12.6 \mathrm{mg} / \mathrm{L} / \mathrm{OD}$ vs. $11.87 \mathrm{mg} / \mathrm{L} / \mathrm{OD})$. The YEF1 overexpressing strain BMY31 resulted in a slight decrease in fatty alcohol production compared to BMY13. These strains BMY30, BMY31 also saw the same slight decrease in cell density associated with the YCpNC1 carrying control strain (BMY13). The decrease in growth associated with these strains may be due to the fact that both NAD kinases and the Pyc1 in the POM cycle are ATP-dependent, and therefore effective generation of NADPH to drive fatty alcohol production will occur at the expense of ATP driven processes. Interestingly, combining the overexpression of POS5c and YCpNC1 into strain BMY32 did not result in synergistic or even additive effects on fatty alcohol production. Fatty alcohol production in BMY32 was less than in the cells expressing either Pos5c alone or the Pyc1, 'Mdh2, sMae1 POM cycle (BMY13). The decrease in fatty alcohol production in this strain is also associated with a reduced cell density suggesting extensive metabolic burden or a negative effect caused by the combination of overexpressed gene products.

\section{Conclusion}

Overexpression of enzymatic isoforms to create POM cycles with transhydrogenase-like activity has the potential to increase product titers from glucose in S. cerevisiae. We found that a POM cycle consisting of Pyc1, 'Mdh2 and sMae1 (YCpNC1) increased fatty alcohol product titers most significantly in two distinct S. cerevisiae strain backgrounds. YCpNC1 resulted in an approximately $40 \%$ increase in fatty alcohol production in our $F A S 1$ overexpressing background and a $27 \%$ increase in our $z w f 1 \triangle$ background. Additionally, overexpression of UTR1 and POS5c can both serve to increase fatty alcohol titers, although the effect of UTR1 is small while overexpression of POS5c is comparable with expression of YCpNC1. Combined overexpression of POS5c and YCpNC1 was found to have detrimental effects particularly in regards to biomass accumulation. These results can likely be applied to increase the availability of $\mathrm{NADPH}$ in many cell systems to enhance biosynthetic processes.

\section{Declarations}

\section{Conflicts of Interest}

The authors declare no conflicts of interest

\section{Ethics Approval and Consent to Participate}

Not applicable

\section{Consent for Publication}

Not applicable

\section{Author Contributions}


DTS and BAM conceived of this study and wrote the paper. CK cloned the single enzyme control strains with myc tags. AK cloned the UTR1 and YEF1 NAD kinases and performed the initial screen for fatty alcohol production using these strains. All other experiments and data analysis were performed by BAM.

\section{Acknowledgements}

We thank XiaoDong Liu for the creation of the FAS1 overexpression strain. This research was supported by grant funding provided by Biolndustrial Innovations Canada (BIC) Bioproducts cluster, Alberta Innovates BioSolutions BFR-16-078 and a Natural Sciences and Engineering Research Council of Canada (NSERC) Discovery grant. BAM was in part supported by an NSERC postdoctoral fellowship.

Availability of data and materials

All data supporting the conclusions of this article are included within the article and its additional files.

\section{References}

1. Akhtar MK, Jones PR: Cofactor engineering for enhancing the flux of metabolic pathways. Front Bioeng Biotechnol 2014, 2:30-30.

2. d'Espaux L, Ghosh A, Runguphan W, Wehrs M, Xu F, Konzock O, Dev I, Nhan M, Gin J, Reider Apel A, et al: Engineering high-level production of fatty alcohols by Saccharomyces cerevisiae from lignocellulosic feedstocks. Metab Eng 2017, 42:115-125.

3. Sheng J, Feng X: Metabolic engineering of yeast to produce fatty acid-derived biofuels: bottlenecks and solutions. Front Microbio/ 2015, 6:554.

4. Jones E, Fink G: Regulation of amino acid and nucleotide biosynthesis in yeast. In The Molecular Biology of the Yeast Saccharomyces: Metabolism and Gene Expression,. Edited by Strathern J, Jones E, Broach J. Cold Spring Harbor, NY: Cold Spring Harbor Laboratory Press; 1982: Pp.181-299.

5. Liu J, Li H, Zhao G, Caiyin Q, Qiao J: Redox cofactor engineering in industrial microorganisms: strategies, recent applications and future directions. J Ind Microbiol Biotechnol 2018, 45:313-327.

6. Spaans S, Weusthuis R, Van Der Oost J, Kengen S: NADPH-generating systems in bacteria and archaea. Front Microbiol 2015, 6:742.

7. Bruinenberg PM: The NADP(H) redox couple in yeast metabolism. Antonie van Leeuwenhoek 1986, 52:411-429.

8. Anderlund M, Nissen TL, Nielsen J, Villadsen J, Rydström J, Hahn-Hägerdal B, Kielland-Brandt MC: Expression of the Escherichia coli pntA and pntB genes, encoding nicotinamide nucleotide transhydrogenase, in Saccharomyces cerevisiae and its effect on product formation during anaerobic glucose fermentation. App/ Environ Microbiol 1999, 65:2333-2340.

9. Nissen TL, Anderlund M, Nielsen J, Villadsen J, Kielland-Brandt MC: Expression of a cytoplasmic transhydrogenase in Saccharomyces cerevisiae results in formation of 2-oxoglutarate due to 
depletion of the NADPH pool. Yeast 2001, 18:19-32.

10. Hou J, Lages NF, Oldiges M, Vemuri GN: Metabolic impact of redox cofactor perturbations in Saccharomyces cerevisiae. Metab Eng 2009, 11:253-261.

11. Liu H, Marsafari $M$, Deng $L, X u P$ : Understanding lipogenesis by dynamically profiling transcriptional activity of lipogenic promoters in Yarrowia lipolytica. App/ Microbiol Biotechno/ 2019, 103:31673179.

12. Moreira dos Santos $M$, Raghevendran V, Kötter $P$, Olsson L, Nielsen J: Manipulation of malic enzyme in Saccharomyces cerevisiae for increasing NADPH production capacity aerobically in different cellular compartments. Metab Eng 2004, 6:352-363.

13. Miyagi H, Kawai S, Murata K: Two sources of mitochondrial NADPH in the yeast Saccharomyces cerevisiae. J Biol Chem 2009, 284:7553-7560.

14. Ida K, Ishii J, Matsuda F, Kondo T, Kondo A: Eliminating the isoleucine biosyntheticpathway to reduce competitive carbon outflow during isobutanol production by Saccharomyces cerevisiae. Microb Cell Fact 2015, 14:62.

15. Matsuda F, Ishii J, Kondo T, Ida K, Tezuka H, Kondo A: Increased isobutanol production in Saccharomyces cerevisiae by eliminating competing pathways and resolving cofactor imbalance. Microb Cell Fact 2013, 12:119.

16. Suga $\mathrm{H}$, Matsuda F, Hasunuma T, Ishii J, Kondo A: Implementation of a transhydrogenase-like shunt to counter redox imbalance during xylose fermentation in Saccharomyces cerevisiae. Appl Microbiol Biotechnol 2013, 97:1669-1678.

17. Yu T, Zhou YJ, Huang M, Liu Q, Pereira R, David F, Nielsen J: Reprogramming Yeast Metabolism from Alcoholic Fermentation to Lipogenesis. Cell 2018, 174:1549-1558.e1514.

18. McAlister-Henn L, Thompson LM: Isolation and expression of the gene encoding yeast mitochondrial malate dehydrogenase. J Bacterio/ 1987, 169:5157-5166.

19. Thompson LM, McAlister-Henn L: Dispensable presequence for cellular localization and function of mitochondrial malate dehydrogenase from Saccharomyces cerevisiae. J Biol Chem 1989, 264:12091-12096.

20. Chen S-J, Wu X, Wadas B, Oh J-H, Varshavsky A: An N-end rule pathway that recognizes proline and destroys gluconeogenic enzymes. Science 2017, 355.

21. Minard KI, McAlisterhenn L: Glucose-Induced Phosphorylation of the MDH2 Isozyme of Malate Dehydrogenase in Saccharomyces cerevisiae. Arch Biochem Biophys 1994, 315:302-309.

22. Minard KI, McAlister-Henn L: Isolation, Nucleotide Sequence Analysis, and Disruption of the MDH2 Gene from Saccharomyces cerevisiae: Evidence for Three Isozymes of Yeast Malate Dehydrogenase. Mol Cell Biol 1991, 11:370-380.

23. Holzer H: Proteolytic catabolite inactivation in Saccharomyces cerevisiae. Revisiones sobre biologia celular: RBC 1989, 21:305-319. 
24. Steffan JS, McAlister-Henn L: Isolation and characterization of the yeast gene encoding the MDH3 isozyme of malate dehydrogenase. J Biol Chem 1992, 267:24708-24715.

25. Brewster NK, Val DL, Walker ME, Wallace JC: Regulation of Pyruvate Carboxylase Isozyme (PYC1, PYC2) Gene Expression in Saccharomyces cerevisiae during Fermentative and Nonfermentative Growth. Arch Biochem Biophys 1994, 311:62-71.

26. Huet C, Menendez J, Gancedo C, François JM: Regulation of pyc1 encoding pyruvate carboxylase isozyme I by nitrogen sources in Saccharomyces cerevisiae. Eur J Biochem 2000, 267:6817-6823.

27. Menéndez J, Gancedo C: Regulatory regions in the promoters of the Saccharomyces cerevisiae PYC1 and PYC2 genes encoding isoenzymes of pyruvate carboxylase. FEMS Microbiol Lett 1998, 164:345352.

28. Shi F, Kawai S, Mori S, Kono E, Murata K: Identification of ATP-NADH kinase isozymes and their contribution to supply of NADP(H) in Saccharomyces cerevisiae. FEBS J 2005, 272:3337-3349.

29. Apps DK: The NAD Kinases of Saccharomyces cerevisiae. Eur J Biochem 1970, 13:223-230.

30. Zhao X, Shi F, Zhan W: Overexpression of ZWF1 and POS5 improves carotenoid biosynthesis in recombinant Saccharomyces cerevisiae. Lett Appl Microbiol 2015, 61:354-360.

31. Paramasivan K, Kumar Hn P, Mutturi S: Systems-based Saccharomyces cerevisiae strain design for improved squalene synthesis. Biochem Eng J 2019, 148:37-45.

32. Paramasivan K, Mutturi S: Regeneration of NADPH Coupled with HMG-CoA Reductase Activity Increases Squalene Synthesis in Saccharomyces cerevisiae. J Agric Food Chem 2017, 65:8162-8170.

33. Navarrete C, Nielsen J, Siewers V: Enhanced ethanol production and reduced glycerol formation in fps1 $1 \Delta$ mutants of Saccharomyces cerevisiae engineered for improved redox balancing. AMB Express 2014, 4:86.

34. Gietz R, Sugino A: New yeast-Escherichia coli shuttle vectors constructed with in vitro mutagenized yeast genes lacking six-base pair restriction sites. Gene 1988, 74:527-534.

35. Gibson DG, Young L, Chuang R-Y, Venter JC, Hutchison CA, Smith HO: Enzymatic assembly of DNA molecules up to several hundred kilobases. Nat Meth 2009, 6:343-345.

36. Ofuonye E, Kutin K, Stuart DT: Engineering Saccharomyces cerevisiae fermentative pathways for the production of isobutanol. Biofuels 2013, 4:185-201.

37. Gietz RD, Woods RA: Transformation of yeast by lithium acetate/single-stranded carrier DNA/polyethylene glycol method. Methods Enzymol 2002, 350:87-96.

38. Foiani M, Marini F, Gamba D, Lucchini G, Plevani P: The B subunit of the DNA polymerase alphaprimase complex in Saccharomyces cerevisiae executes an essential function at the initial stage of DNA replication. Mol Cell Biol 1994, 14:923.

39. Schmitt ME, Brown TA, Trumpower BL: A rapid and simple method for preparation of RNA from Saccharomyces cerevisiae. Nucleic Acids Res 1990, 18:3091-3092.

40. Aranda PS, LaJoie DM, Jorcyk CL: Bleach Gel: A Simple Agarose Gel for Analyzing RNA Quality. Electrophoresis 2012, 33:366-369. 
41. Boles E, De Jong-Gubbels P, Pronk JT: Identification and characterization of MAE1, the Saccharomyces cerevisiae structural gene encoding mitochondrial malic enzyme. J Bacterio/1998, 180:2875-2882.

42. Jitrapakdee S, Adina-Zada A, Besant PG, Surinya KH, Cleland WW, Wallace JC, Attwood PV: Differential regulation of the yeast isozymes of pyruvate carboxylase and the locus of action of acetyl CoA. Int J Biochem Cell Biol 2007, 39:1211-1223.

43. Schüller HJ, Hahn A, Tröster F, Schütz A, Schweizer E: Coordinate genetic control of yeast fatty acid synthase genes FAS1 and FAS2 by an upstream activation site common to genes involved in membrane lipid biosynthesis. EMBO J 1992, 11:107-114.

\section{Figures}

A

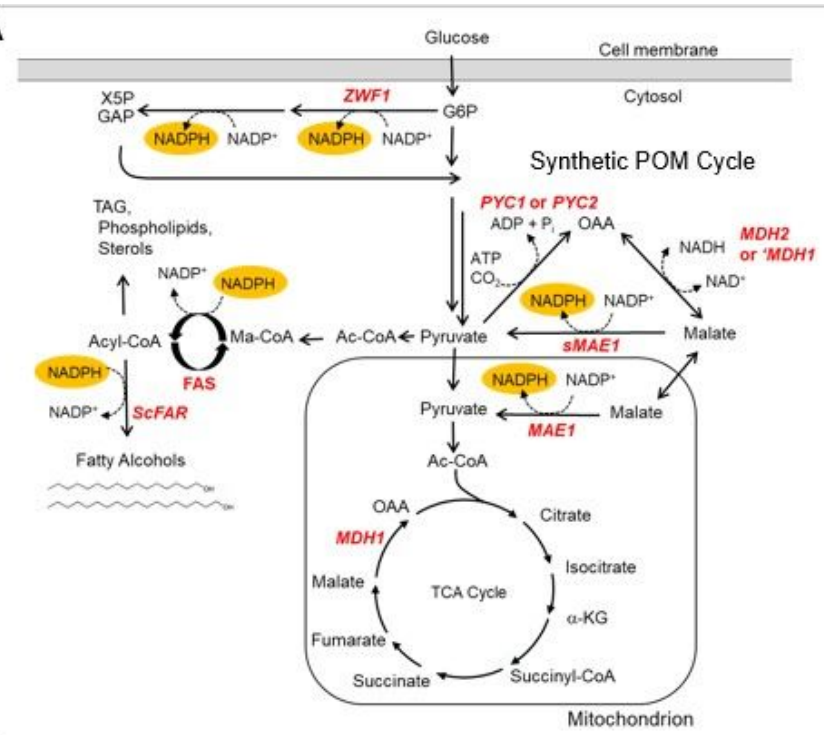

B

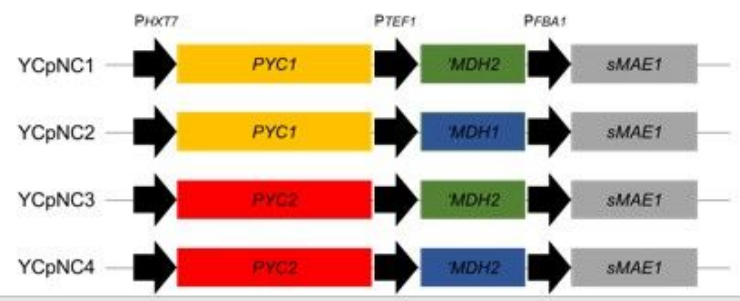

Figure 1.

Figure 1

Synthetic POM cycles in the context of cellular metabolic pathways. The schematic illustrates the native roles and localizations of the enzymes utilized in the synthetic POM cycles as well as the proposed localization of the modified versions 'Mdh1 and sMae1 (A). Production of NADPH through the pentose phosphate pathway is noted as is the consumption of NADPH in the production of fatty alcohols, through the overexpression of the fatty acyl synthetase complex (FAS) and the heterologous expression of fatty 
acyl-CoA reductase (FAR). The composition of each of the plasmids containing synthetic POM cycles is shown in (B). Abbreviations in part (A) of the diagram: G6P Glucose-6-phosphate, X5P ribulose 5 phosphate, GAP glyceraldehyde 3-phosphate, OAA oxaloacetate, Ac-CoA acetyl-CoA, Ma-CoA malonyl CoA, a-KG alpha ketoglutarate, TAG triacyclglycerols.

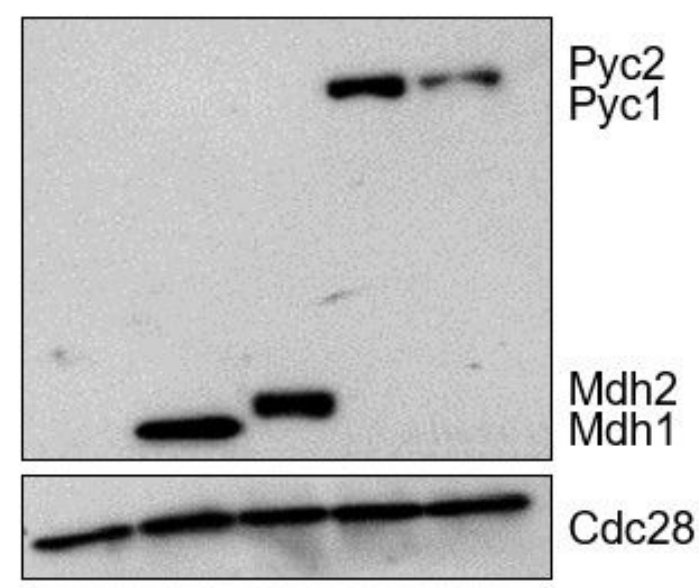

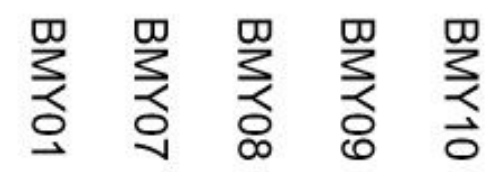

Figure 2

Verification of protein expression of 'Mdh1, 'Mdh2, Pyc1 and Pyc2 by western blot using the 『-MYC antibody. Lower panel, loading control blotting for Cdc28 with the a-PSTAIRE antibody. 
A

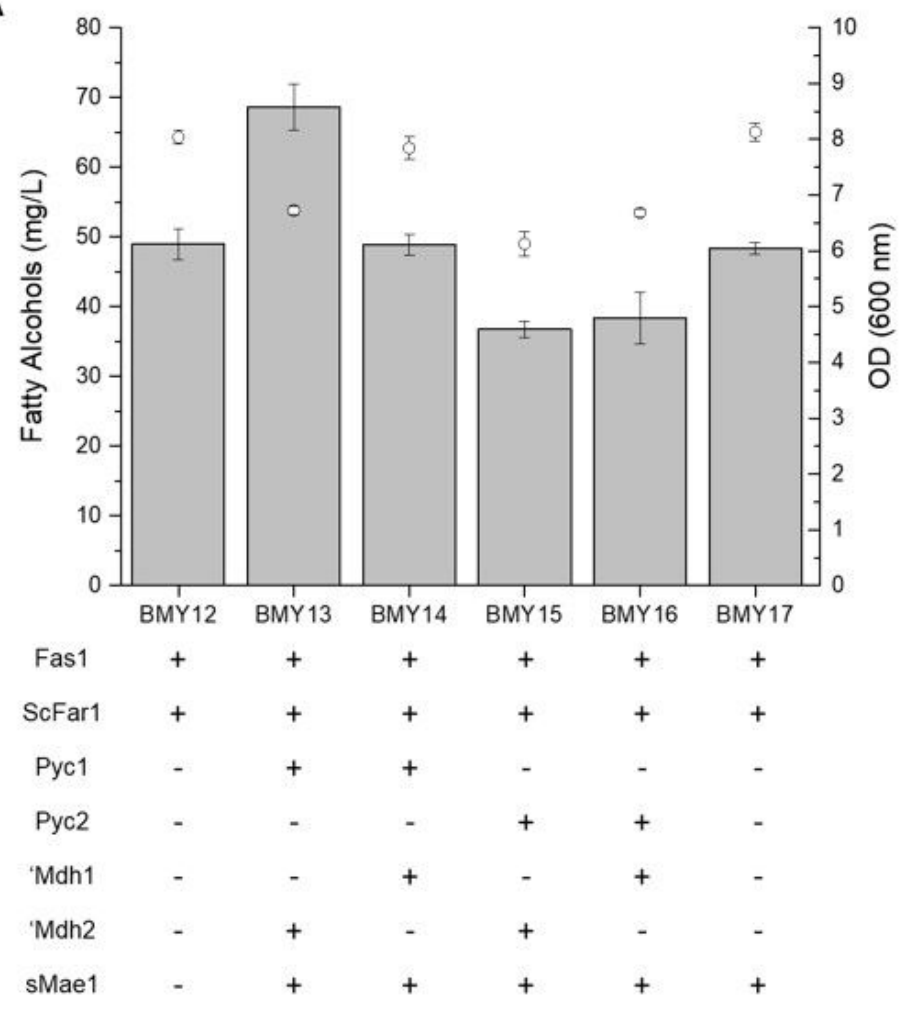

B

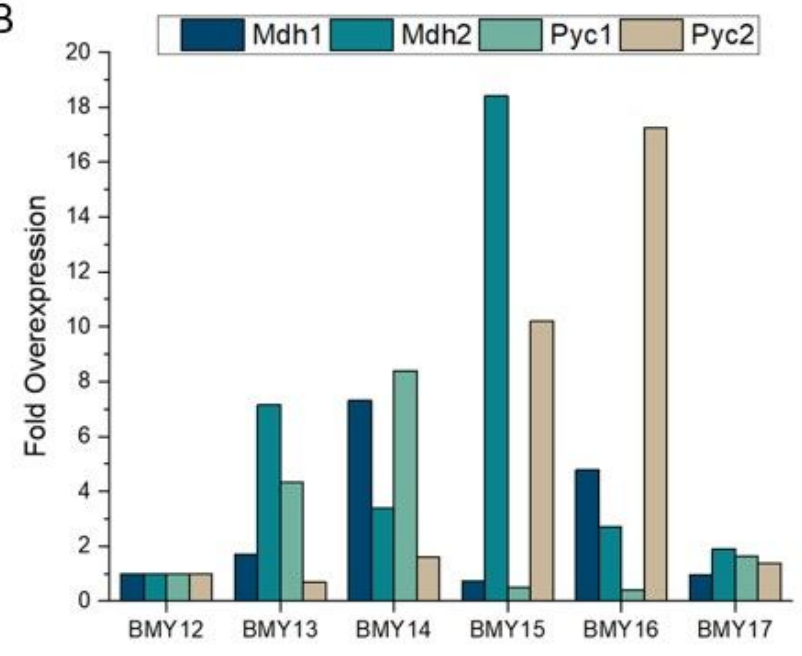

$\begin{array}{llllllll}\text { Fas1 } & + & + & + & + & + & + & \\ \text { ScFar1 } & + & + & + & + & + & + & \\ \text { Pyc1 } & - & + & + & - & - & - & \\ \text { Pyc2 } & - & - & - & + & + & - & \\ \text { Mdh1 } & - & - & + & - & + & - & \\ \text { Mdh2 } & - & + & - & + & - & - & \\ \text { SMae1 } & - & + & + & + & + & + & \text { Figure 3. }\end{array}$

Figure 3

Effects of synthetic POM cycles in FAS overexpressing, fatty alcohol producing strains. (A) Shaded bars indicate mean fatty alcohol titers; open circles indicate mean cell density (OD600) after 72 hours of shake flask culture. Error bars indicate standard deviation. The data corresponding to individual cultures is recorded in Fig S3. (B) mRNA corresponding to MDH1, MDH2, PYC1, PYC2 from the POM cycle expressing strains determined by RT-qPCR. 


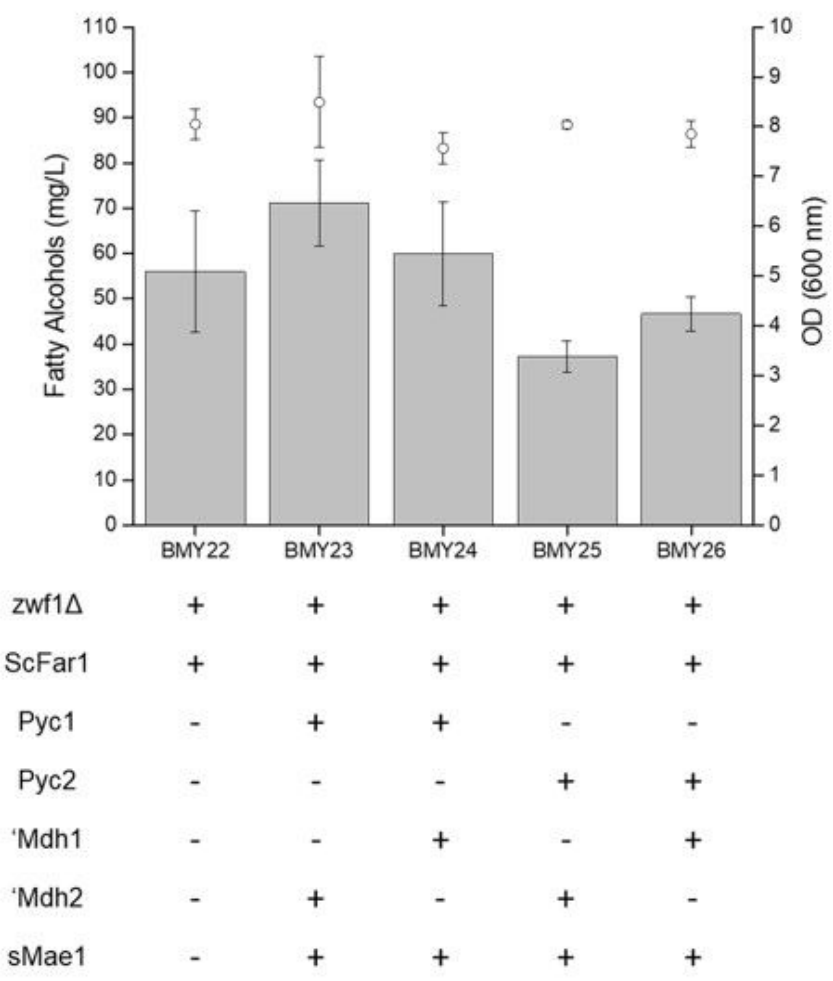

Figure 4

\section{Figure 4}

Effect of the POM cycles on fatty alcohol production in a zwf1 knockout strain. Mean fatty alcohol production (mg/L) and OD600 after 72 hours are shown for three independent biological replicates of strains BMY22-BMY26 (open circles). 


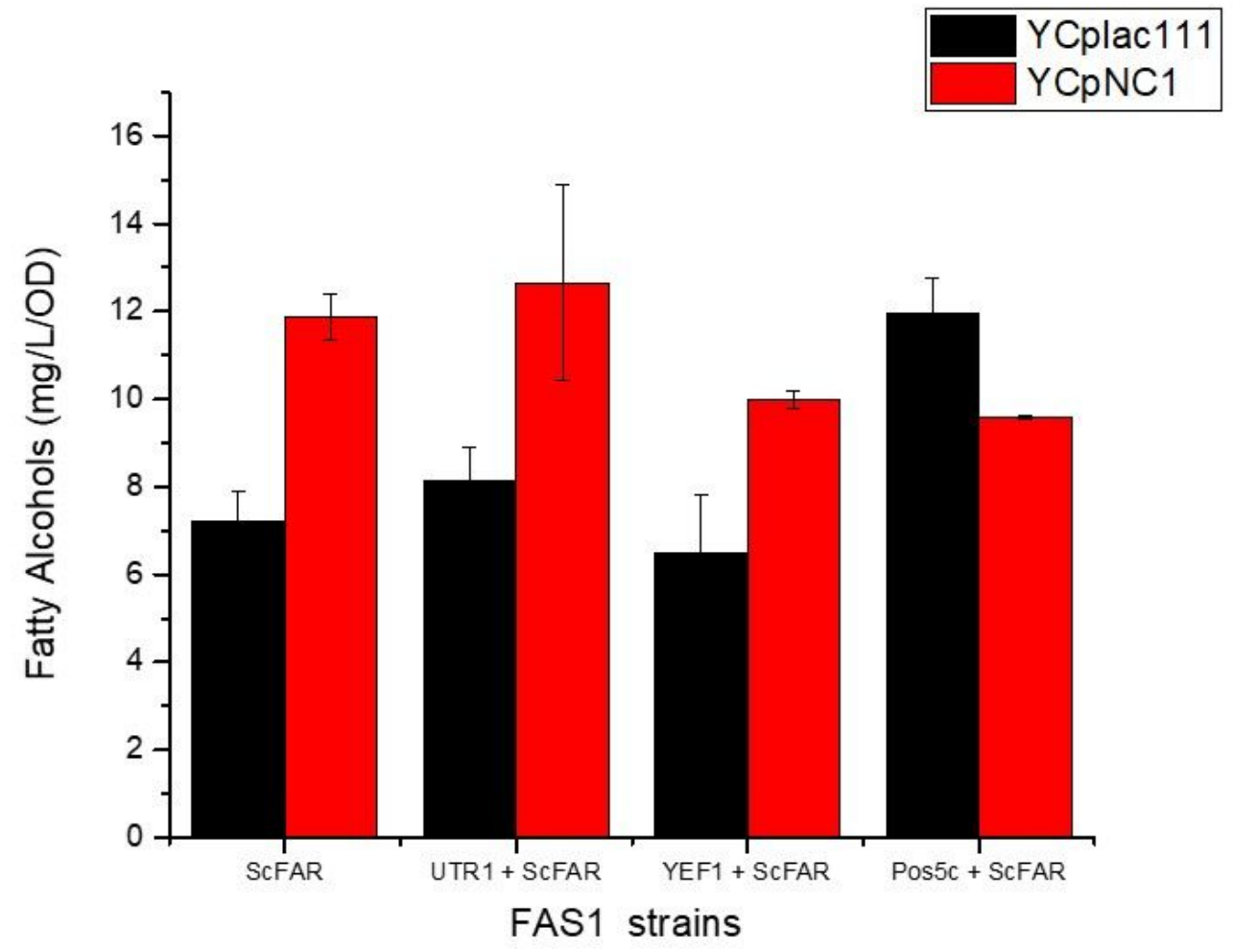

Figure 5.

Figure 5

Effect of the NAD Kinases Utr1, Yef1, and Pos5c on fatty alcohol production. Fatty alcohol titers (mg/L/OD) for high producers of strains expressing NAD kinases with or without co-expression of the POM cycle encoded by YCpNC1. Data for original biological replicates can be seen in Figure S7.

\section{Supplementary Files}

This is a list of supplementary files associated with this preprint. Click to download.

- SupplementalsyntheticPOMcycles1162020.docx 